\title{
Temporal trends in the availability and efficacy of catheter ablation for atrial fibrillation and atrial flutter in a highly populated urban area
}

\author{
Maciej Faryan', Piotr Buchta², Oskar Kowalski³, Maciej T. Wybraniec², Daniel Cieśla², Krzysztof Myrda², \\ Anna Wnuk-Wojnar¹, Zbigniew Kalarus³ ${ }^{3}$ Mariusz Gąsior², Katarzyna Mizia-Stec ${ }^{1}$ \\ 1 1st Department of Cardiology, School of Medicine in Katowice, Medical University of Silesia, Upper Silesia Medical Center, Katowice, Poland \\ 2 3rd Department of Cardiology, School of Medicine with the Division of Dentistry in Zabrze, Medical University of Silesia, Silesian Center for Heart Diseases, Zabrze, Poland \\ 3 Department of Cardiology, Congenital Heart Diseases and Electrotherapy, School of Medicine with the Division \\ of Dentistry in Zabrze, Medical University of Silesia, Silesian Center for Heart Diseases, Zabrze, Poland
}

\section{KEY WORDS}

atrial fibrillation, catheter ablation, pulmonary vein isolation, registry
Correspondence to: Prof. Katarzyna Mizia-Stec, MD, PhD, FESC, 1st Department of Cardiology, School of Medicine in Katowice, Medical University of Silesia, Upper Silesia Medical Center, ul. Ziołowa 47, 40-635 Katowice, Poland, phone: +48323598890 , email: kmizia@op.pl Received: January 20, 2020. Revision accepted: April 2, 2020 Published online: April 2, 2020. Kardiol Pol. 2020; 78 (6): 537-544 doi:10.33963/KP.15275 Copyright by the Author(s), 2020

\begin{abstract}
BACKGROUND Pulmonary vein isolation has become one of the core modalities of the rhythm control strategy in patients with atrial fibrillation (AF).

AIMS The aim of the study was to analyze temporal trends in the availability and efficacy of AF and atrial flutter (AFL) catheter ablation in an urban area of Upper Silesia in Poland.

METHODS The source data were obtained from the SILCARD (Silesian Cardiovascular Database) covering an adult population of 3.8 million. The final study population included patients with diagnosis code I48 referred for catheter ablation between 2006 and 2017. The data included total number of procedures, patient sex, age, comorbidities, number of hospital admissions, and mortality rate.

RESULTS A total of 2745 patients were enrolled. The number of ablated patients increased more than 10 -fold (43 in 2006 vs 507 in 2017; $P=0.008$ ) in the follow-up period. The analysis showed an upward trend in the proportion of women $(P=0.02)$, hypertension prevalence $(P=0.004)$, and percentage of patients implanted $(P=0.02)$. A decrease was observed in the percentage of patients with stable angina $(P<0.005)$ and hospitalization length $(P<0.005)$. The all-cause hospital readmissions rate decreased from $55.8 \%$ to $25.4 \%(P<0.005)$. There were significant reductions in the 12 -month all-cause mortality $(2.3 \%$ in 2006 vs $0.2 \%$ in $2017 ; P<0.005)$, stroke ( $2.3 \%$ in 2006 vs $0.2 \%$ in $2017 ; P=0.047)$, and myocardial infarction rates ( $2.3 \%$ in 2006 vs $0.4 \%$ in $2017 ; P=0.03$ ).

CONCLUSIONS A considerable increase in the availability and efficacy of AF/ AFL ablations was documented over the 12-year follow-up period.
\end{abstract}

INTRODUCTION Atrial fibrillation (AF) is one of the leading causes of cardiovascular hospitalizations worldwide. Affecting more than 3\% of the general adult population, ${ }^{1} \mathrm{AF}$ is accountable for nearly $25 \%$ of all ischemic strokes and stroke-related mortality. ${ }^{2}$ Despite a considerable AF-related healthcare burden, only treatment with oral anticoagulants has shown unequivocal impact on mortality reduction, while both rate- and rhythm-control strategies exerted comparable effects on long-term outcomes. ${ }^{3}$
Still, sinus rhythm maintenance is often pursued in young patients with short history of arrhythmic episodes and small comorbidity burden. This rhythm-control strategy includes antiarrhythmic drugs, electrical cardioversion, and circumferential pulmonary vein isolation (PVI). ${ }^{4}$ Since the proposal of Haïssaguerre's theory regarding ectopic beats originating from the pulmonary veins in the late $1990 \mathrm{~s},{ }^{5}$ the technique of AF ablation has substantially evolved into one of the mainstays of antiarrhythmic therapy. ${ }^{6}$ 


\section{WHAT'S NEW?}

Although catheter ablation for atrial fibrillation has emerged as a widely accessible intervention, the exact annual number of procedures and in-hospital and long-term mortality rates are unknown. The presented registry-based data demonstrated an increasing annual number of catheter ablation procedures for atrial fibrillation and atrial flutter in a large and highly populated area in central Europe. The growing number of interventions was accompanied by relatively low in-hospital and 1-year mortality rates of patients with atrial fibrillation / atrial flutter referred for percutaneous catheter ablation. These results deliver evidence for relative short- and long-term safety of pulmonary vein isolation.
Fundusz Zdrowia; NFZ), which provided all data for the database, covering the period between 2006 and 2017.

The SILCARD registry-based database enrolled all consecutive Silesian adult patients hospitalized in cardiology, cardiac surgery, vascular surgery or diabetology units for any reason, or hospitalized in the internal medicine or intensive care units with the principal diagnosis of cardiovascular disease or with the diagnosis of stroke at the neurology department. ${ }^{13}$ Cardiovascular disease was defined as code R52 or J96 or any I code according to the International Statistical Classification of Diseases, Tenth Revision (ICD-10).

Altough PVI leads to symptomatic impro ment in about $50 \%$ to $70 \%$ of patients, ${ }^{7,8}$ the impact of PVI on hard clinical endpoints is less certain and has so far been confirmed only in patients with systolic heart failure. ${ }^{9}$

Considering its high procedural costs and the prerequisite qualification process, the rate of PVI procedures may be treated as a surrogate for quality of cardiovascular care. According to the recent results of the AF registry from the European Observational Research Programme, the utilization of PVI in academic centers in Poland was as high as $13.9 \%{ }^{10}$; however, the exact clinical characteristics, as well as in-hospital morbidity and mortality of this population is unknown. Former data indicated a rapid surge in the number of AF ablation procedures in Europe, ${ }^{11,12}$ but the precise data on short- and long-term outcomes, including mortality, are scarce.

The present study presents the data acquired from the Silesian Cardiovascular Registry (SILCARD) established in this region of Upper Silesia, Poland, which collects in-hospital and follow-up data regarding all hospital admissions in medical centers cooperating with the single national healthcare provider. The primary aim was to characterize the population of patients with AF and/or atrial flutter (AFL) subject to PVI or cavotricuspid isthmus ablation with special consideration of the prevalence of comorbidities, length of hospitalization as well as in-hospital and 12-month morbidity and mortality.

METHODS Data source General information on the SILCARD (ClinicalTrials.gov identifier, NCT02743533) database was reported previously. ${ }^{13}$ Briefly, the database contains records from all hospitals $(n=310)$ in the Silesian Province-a large administrative region in southern Poland with a population of 4.57 million (roughly $12 \%$ of Poland's total population) of which 3.8 million are adults. The Silesian Province provides a well-developed hospital network with 2 tertiary cardiology hospitals, 3 cardiac surgery departments, and 20 catheterization laboratories. The only healthcare provider in Poland is the National Health Fund (Narodowy
Study population The analysis included all patients from the SILCARD database who underwent the catheter ablation with the principal diagnosis coded according to the ICD-10 as I48 (AF or AFL) between 2006 and 2017. The study flow-chart is shown in FIGURE 1 . Data on all individual patients and all hospitalizations were analyzed. Patients who underwent the ablation procedure were classified according to procedural codes from the ICD-9 (International Classification System for Surgical, Diagnostic and Therapeutic Procedures): 37.341, 37.342, 37.272 (for 3-dimensional [3D] mapping), and 37.261 (for electrophysiological study). The inclusion criterion involved patients with both AF and AFL subject to catheter ablation with electroanatomical mapping using 3D navigation system. According to the NFZ's reimbursement policy, both PVI and cavotricuspid isthmus ablation fall under the same reporting category, and therefore, both procedures cannot be distinguished based on the SILCARD database. Yet, the proportion of patients with AFL was derived from local data from one of the biggest ablation centers in Upper Silesia. Cavotricuspid isthmus ablation for AFL with 3D mapping was performed in 74 patients $(8.5 \%)$ out of the total of 874 procedures.

The exclusion criteria included: 1 ) residency outside the Silesian Province, 2) age younger than 18 years at the time of ablation, 3) history of any ablation within the preceding year, 4) a simple ablation procedure (eg, atrioventricular node ablation) or electrophysiological study only without ablation defined with NFZ unit product codes 5.06.00.0000969, 5.51.01.0005 044, and 5.06.00.0000970.

Study data Data available from the NFZ included the total number of patients ablated with the primary AF diagnosis, their sex, age, comorbidities, hospitalizations, and mortality. All the information was gathered anonymously so that individual cases could not be identified. Patients signed informed consent prior to participation in the study, which involved enrolment to NFZ registries. The SILCARD registry 


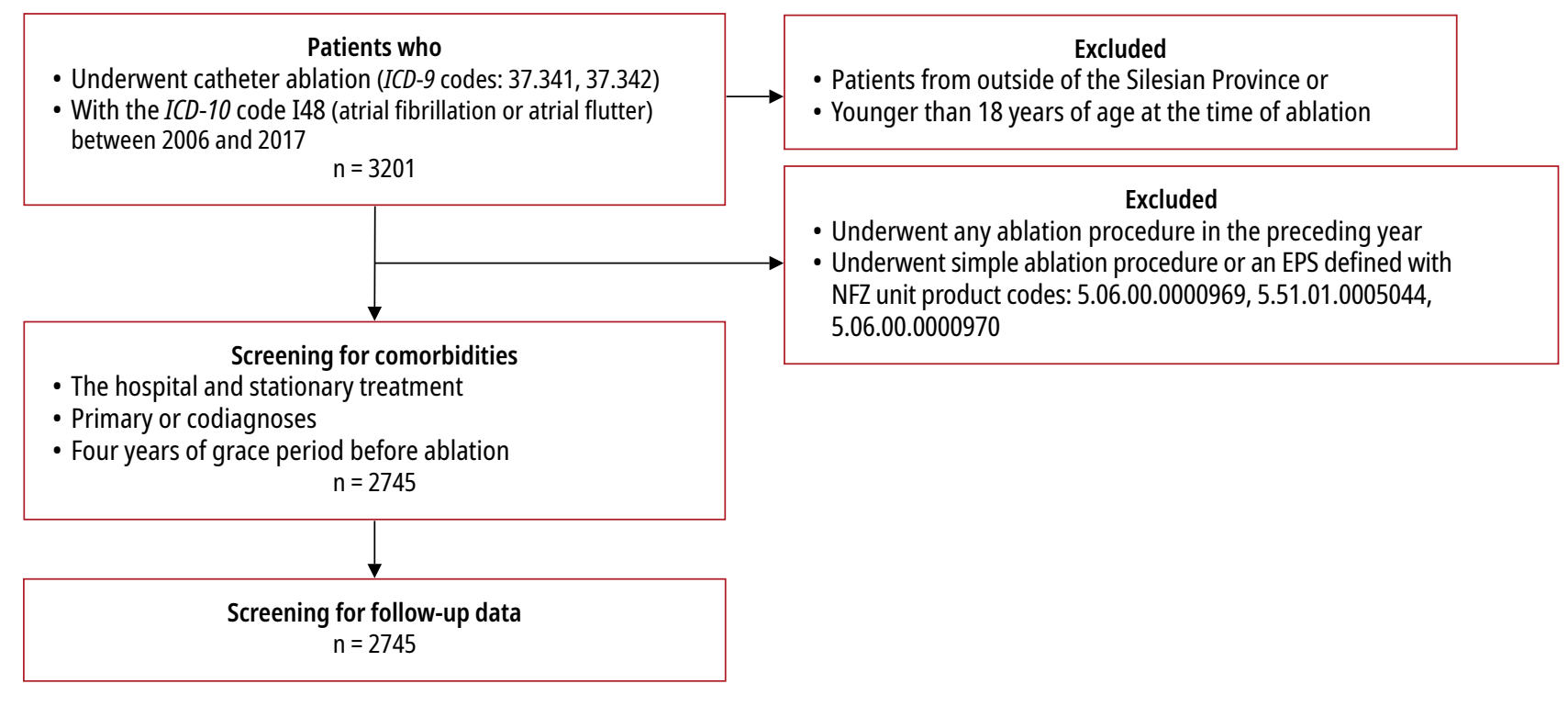

FIGURE 1 Study flow-chart

Abbreviations: EPS, electrophysiology study; ICD-9, International Statistical Classification of Diseases, Ninth Revision; ICD-10, International Statistical Classification of Diseases, Tenth Revision; NFZ, the Polish National Health Fund

was approved by the ethics committee of Medical University of Silesia and was performed according to the ethical standards laid down in the 1964 Declaration of Helsinki and its later amendments.

In general, every medical consultation or hospitalization in Poland is reported to the NFZ using dates of care and the principal diagnosis and up to 2 comorbidities defined with an ICD-10 code key. The screening for comorbidities was based on ICD-10 and ICD-9 codes relayed from hospitals and outpatient specialist clinics and general practitioner clinics. In order to avoid exclusion of rare concomitant diseases, the analysis was performed after 1 year of grace period before ablation.

Data on the following conditions were obtained: hypertension (ICD-10: I1*), stable angina (ICD-10: I25*, I20.1, I20.8, I20.9), history of myocardial infarction (MI; ICD-10: I21-I22), heart failure (ICD-10: I50, I42), diabetes (ICD-10: E10-E14), chronic kidney disease (CKD, ICD-10: N17-19), chronic obstructive pulmonary disease (ICD-10: J44), stroke (ICD-10: I60-I64), hyperlipidemia (ICD-10: E78), thyroid function disorders (ICD-10: E01, E02, E03, E05), history of valvular surgery (ICD-9: 35.0, 35.1, 35.2, 35.3) as well as pacemaker (ICD-9: 37.8) /implantable cardioverter-defibrillator (ICD-9: 37.941-944, 37.961, 37.962, 37.991) / cardiac resynchronization therapy (CRT; ICD-9: 00.50, 00.51, 00.53, 0054).

If a patient was transferred to another department or hospital or the time between hospital discharge and the following admission due to the diagnosed CV disease was shorter than 1 day, all following hospitalizations were merged into a single hospital stay.

The collected follow-up data included: 1) length of hospital stays, 2) in-hospital mortality, 3) all-cause hospital readmissions, 4) hospital readmissions due to cardiovascular reason (with any principal diagnosis of cardiovascular disease), 5) hospital readmissions due to AF, 6) hospital readmissions due to heart failure, 7) stroke, 8) cardiac cardioversion (ICD-9: 99.62*, 99.622, 99.624, 99.61), 9) bleeding complications (ICD-10: I84*, I85.0, K22.6 or ICD-9 code $99.0^{*}$ for blood transfusion), 10) MI, and 11) all-cause mortality in a 12-month follow-up.

Statistical analysis Statistical analysis was performed using SPSS software version 25.0 (IBM Corp., Armonk, New York, United States). The qualitative variables were expressed as absolute number and percentage, while continuous variables as mean and SD. The distribution of continuous variables was verified using the Shapiro-Wilk test. The temporal trends for continuous variables were verified using a 2-way analysis of variance (ANOVA) or repeated measures ANOVA if applicable. A $P$ value of less than 0.05 was regarded as significant.

RESULTS The temporal trends in demographic and clinical characteristics are presented in tABlE 1. The data concerning the number and duration of hospitalization are presented in TABLE 2. The trends in adverse events and medical contacts in the registry population in a 12-month follow-up period are shown in TABLE3.

A total of 2745 patients with the ICD-10 code 148 were referred for catheter ablation. The number of ablations increased more than 10 -fold (43 in 2006 vs 507 in 2017; $P=0.008$; 133 / 1 million in 2017; FIGURE 2), and the mean age of patients undergoing the procedure 
TABLE 1 Baseline characteristics and comorbidities of patients ablated with the principal diagnosis of atrial fibrillation / flutter

\begin{tabular}{|c|c|c|c|c|c|c|c|c|c|c|c|c|c|}
\hline & 2006 & 2007 & 2008 & 2009 & 2010 & 2011 & 2012 & 2013 & 2014 & 2015 & 2016 & 2017 & $\begin{array}{l}P \text { value } \\
\text { for } \\
\text { trend }\end{array}$ \\
\hline Patients, $\mathrm{n}$ & 43 & 54 & 72 & 119 & 157 & 159 & 202 & 328 & 348 & 407 & 349 & 507 & - \\
\hline Age, y, mean (SD) & $\begin{array}{l}58.47 \\
(12.71)\end{array}$ & $\begin{array}{l}56.94 \\
(12.58)\end{array}$ & $\begin{array}{l}59.33 \\
(11.58)\end{array}$ & $\begin{array}{l}57.81 \\
(11.25)\end{array}$ & $\begin{array}{l}59.43 \\
(11.67)\end{array}$ & $\begin{array}{l}58.06 \\
(11.35)\end{array}$ & $\begin{array}{l}59.23 \\
(10.73)\end{array}$ & $\begin{array}{l}60 \\
(10.09)\end{array}$ & $\begin{array}{l}60.34 \\
(10.05)\end{array}$ & $\begin{array}{l}60.79 \\
(11.07)\end{array}$ & $\begin{array}{l}60.57 \\
(11.33)\end{array}$ & $\begin{array}{l}60.89 \\
(10.67)\end{array}$ & 0.008 \\
\hline Sex (female) & 27.9 & 20.4 & 33.3 & 31.9 & 31.2 & 30.8 & 23.8 & 31.7 & 33.1 & 40.1 & 33 & 35.3 & 0.02 \\
\hline Hypertension & 27.9 & 57.4 & 56.9 & 54.6 & 58.6 & 62.3 & 53.5 & 52.1 & 56.9 & 51.4 & 45.9 & 46.9 & 0.004 \\
\hline Stable angina & 65.1 & 74.1 & 62.5 & 63.9 & 66.2 & 59.8 & 64.4 & 58.5 & 50 & 45.2 & 36.7 & 36.7 & $<0.005$ \\
\hline History of MI & 9.3 & 3.7 & 4.2 & 4.2 & 3.2 & 1.3 & 5 & 3.4 & 3.5 & 3 & 2.3 & 3.6 & 0.23 \\
\hline Heart failure & 14 & 16.7 & 26.4 & 21.9 & 24.8 & 20.8 & 21.8 & 25.6 & 20.4 & 18.2 & 17.8 & 20.1 & 0.19 \\
\hline Diabetes mellitus & 4.7 & 13 & 13.9 & 18.5 & 12.7 & 12.6 & 14.9 & 15.6 & 15.5 & 14.3 & 12.9 & 17.2 & 0.22 \\
\hline Hyperlipidemia & 0 & 11.1 & 5.6 & 4.2 & 6.4 & 5 & 9.9 & 11.9 & 7.8 & 8.6 & 4 & 7.5 & 0.7 \\
\hline CKD & 2.3 & 0 & 0 & 1.7 & 0.6 & 2.5 & 2 & 2.1 & 1.4 & 2.7 & 2 & 2.6 & 0.1 \\
\hline COPD & 7 & 0 & 2.8 & 2.5 & 5.7 & 1.9 & 5.5 & 4.9 & 4.6 & 4.9 & 5.4 & 5.1 & 0.13 \\
\hline History of stroke & 2.3 & 3.7 & 2.8 & 0.8 & 3.2 & 1.3 & 2 & 2.1 & 0.6 & 2 & 2.3 & 1.8 & 0.58 \\
\hline $\begin{array}{l}\text { Thyroid function } \\
\text { disorders }\end{array}$ & 2.3 & 16.7 & 12.5 & 10.1 & 10.2 & 12.6 & 10.4 & 9.8 & 8.6 & 6.1 & 7.2 & 9.1 & 0.02 \\
\hline $\begin{array}{l}\text { History of valvular } \\
\text { surgery }\end{array}$ & 2.3 & 1.9 & 2.8 & 0 & 1.3 & 1.3 & 1.5 & 0.6 & 0.3 & 0.5 & 0.3 & 0.6 & 0.01 \\
\hline $\begin{array}{l}\text { Pacemaker } \\
\text { implanted }\end{array}$ & 0 & 0 & 5.6 & 3.4 & 3.8 & 3.8 & 1.5 & 2.7 & 3.2 & 1.2 & 1.7 & 1.2 & 0.02 \\
\hline ICD placed & 0 & 0 & 0 & 4.2 & 0 & 1.9 & 0.5 & 2.7 & 1.7 & 1 & 0.9 & 1 & 0.66 \\
\hline CRT implanted & 0 & 0 & 1.4 & 4.2 & 5.1 & 3.8 & 2.5 & 2.1 & 3.2 & 1.5 & 1.2 & 0.8 & 0.007 \\
\hline
\end{tabular}

Data are presented as percentage unless otherwise indicated.

Abbreviations: CKD, chronic kidney disease; COPD, chronic obstructive pulmonary disease; CRT, cardiac resynchronization therapy; HF, heart failure; ICD, implantable cardioverter-defibrillator; MI, myocardial infarction

TABLE 2 Ablation-related hospitalization characteristics of patients ablated with the principal diagnosis of atrial fibrillation / flutter

\begin{tabular}{|c|c|c|c|c|c|c|c|c|c|c|c|c|c|}
\hline & 2006 & 2007 & 2008 & 2009 & 2010 & 2011 & 2012 & 2013 & 2014 & 2015 & 2016 & 2017 & $\begin{array}{l}\text { Pvalue } \\
\text { for } \\
\text { trend }\end{array}$ \\
\hline Patients, $\mathrm{n}$ & 43 & 54 & 72 & 119 & 157 & 159 & 202 & 328 & 348 & 407 & 349 & 507 & - \\
\hline $\begin{array}{l}\text { Length of } \\
\text { hospitalization, d, } \\
\text { mean (SD) }\end{array}$ & $\begin{array}{l}7.07 \\
(4.11)\end{array}$ & $7(4.3)$ & $\begin{array}{l}6.21 \\
(4.85)\end{array}$ & $\begin{array}{l}5.61 \\
(3.03)\end{array}$ & $\begin{array}{l}6.57 \\
(4.51)\end{array}$ & $\begin{array}{l}6.01 \\
(3.89)\end{array}$ & $5.53(3)$ & $\begin{array}{l}5.78 \\
(3.64)\end{array}$ & $\begin{array}{l}4.97 \\
(2.46)\end{array}$ & $\begin{array}{l}5.4 \\
(3.52)\end{array}$ & $\begin{array}{l}5.08 \\
(3.68)\end{array}$ & $\begin{array}{l}4.91 \\
(2.71)\end{array}$ & $<0.005$ \\
\hline $\begin{array}{l}\text { Hospitalization } \geq 7 \\
\text { days, } \%\end{array}$ & 39.5 & 48.2 & 27.8 & 26.1 & 29.9 & 25.2 & 17.8 & 19.2 & 10.9 & 17.9 & 13.2 & 12.4 & $<0.005$ \\
\hline In-hospital death, $\%$ & 0 & 0 & 0 & 0 & 0 & 0 & 0 & 0 & 0 & 0.3 & 0 & 0 & 0.63 \\
\hline
\end{tabular}

increased significantly $(P=0.008$; FIGURE 3$)$. During the follow-up period, the prevalence of stable angina decreased from $65.1 \%$ in 2006 to $36.7 \%$ in 2017 ( $P<0.005)$, whereas that of heart failure, diabetes mellitus, chronic kidney disease, and chronic obstructive pulmonary disease remained stable (TABLE 1). The analysis revealed an upward trend in the prevalence of arterial hypertension $(P=0.004)$ and percentage of patients with implanted cardiac device (pacemaker, $P=0.02 ; C R T, P=0.007)$. Despite no statistical significance in annual fluctuations, the prevalence of stroke remained low during the study period, reaching the highest level of $3.7 \%$ in 2007 and the lowest of $0.6 \%$ in 2014 (TABLE 1).

The analysis of index hospitalization data denoted that the in-hospital mortality rate was extremely low, as only one death occurred in 2015 (0.3\%). The mean (SD) duration of 
TABLE 3 Trends for adverse events and medical consults in the 12-month follow-up in patients ablated with principal diagnosis of atrial fibrillation or atrial flutter (since hospital discharge)

\begin{tabular}{|c|c|c|c|c|c|c|c|c|c|c|c|c|c|}
\hline & 2006 & 2007 & 2008 & 2009 & 2010 & 2011 & 2012 & 2013 & 2014 & 2015 & 2016 & 2017 & $\begin{array}{l}P \text { value } \\
\text { for } \\
\text { trend }\end{array}$ \\
\hline All-cause hospital readmissions & 55.8 & 64.8 & 47.2 & 58.8 & 63.1 & 52.2 & 43.1 & 46.7 & 45.4 & 46.7 & 43.8 & 25.4 & $<0.005$ \\
\hline $\begin{array}{l}\text { Hospital readmission due to a cardiovascular } \\
\text { reason }\end{array}$ & 44.2 & 63 & 44.4 & 47.9 & 54.1 & 40.9 & 32.7 & 34.8 & 29 & 30 & 27.5 & 16.2 & $<0.005$ \\
\hline Hospital readmission due to $\mathrm{AF}$ & 23.3 & 44.4 & 29.2 & 30.3 & 31.2 & 25.8 & 19.8 & 18.6 & 16.4 & 19.2 & 15.5 & 9.7 & $<0.005$ \\
\hline Hospital readmission due to HF & 7 & 5.6 & 6.9 & 8.4 & 13.4 & 4.4 & 7.4 & 7.6 & 5.2 & 3.9 & 5.7 & 3.4 & $<0.005$ \\
\hline Outpatient visits - cardiology, n/year & 3.79 & 3.33 & 3.75 & 3.8 & 2.92 & 2.78 & 2.23 & 2.52 & 2.28 & 2.16 & 2.06 & 0.92 & $<0.005$ \\
\hline $\begin{array}{l}\text { Outpatient visits - general practitioner, } \\
\text { n/year }\end{array}$ & 12.67 & 10.43 & 9.88 & 10.4 & 10.94 & 10.7 & 11.78 & 10.72 & 11.04 & 11.55 & 10.49 & 5.1 & $<0.005$ \\
\hline Reablation & 2.3 & 18.5 & 4.2 & 8.4 & 9.6 & 9.4 & 5 & 8.5 & 8.1 & 4.4 & 5.4 & 2.8 & $<0.005$ \\
\hline Cardioversion & 9.3 & 11.1 & 8.3 & 7.6 & 5.1 & 6.9 & 4 & 4.3 & 4.6 & 5.7 & 6.3 & 3.2 & 0.005 \\
\hline $\begin{array}{l}\text { Gastrointestinal bleeding or blood } \\
\text { transfusion }\end{array}$ & 2.3 & 3.7 & 1.4 & 1.7 & 3.8 & 4.4 & 2 & 2.7 & 1.7 & 2.2 & 2.9 & 2.2 & 0.54 \\
\hline MI & 2.3 & 1.9 & 0 & 0.8 & 1.9 & 0.6 & 1.5 & 0.3 & 0 & 0 & 1.2 & 0.4 & 0.03 \\
\hline Stroke & 2.3 & 0 & 2.8 & 0.8 & 0.6 & 1.9 & 2 & 1.2 & 0.6 & 0.7 & 1.4 & 0.2 & 0.047 \\
\hline All-cause mortality & 2.3 & 1.9 & 0 & 3.4 & 6.4 & 2.5 & 3.5 & 3.4 & 1.4 & 1.2 & 0.6 & 0.2 & $<0.005$ \\
\hline
\end{tabular}

Data are presented as percentage unless otherwise indicated.

Abbreviations: see TABLE 1

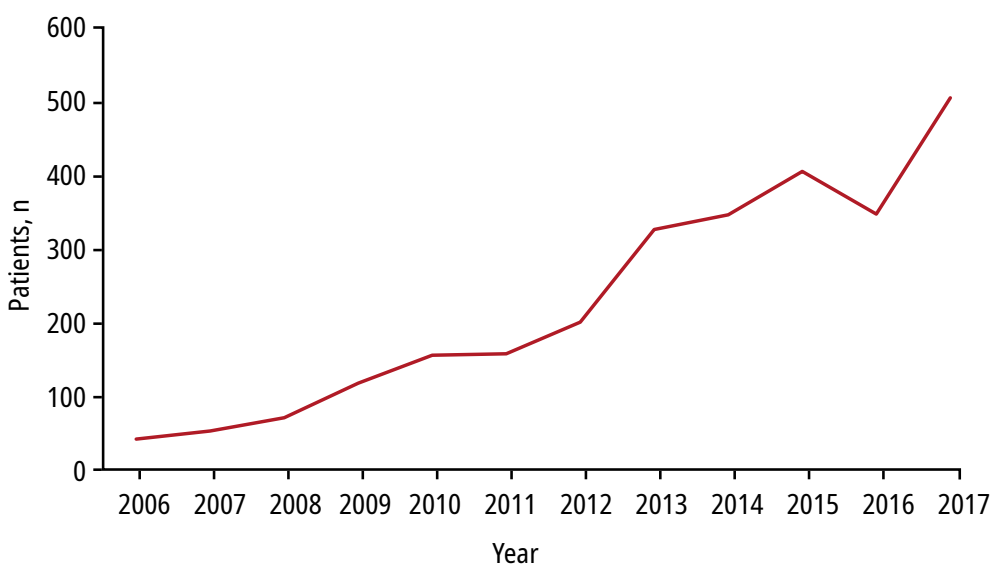

FIGURE 2 Annual number of patients undergoing pulmonary vein isolation or cavotricuspid isthmus ablation

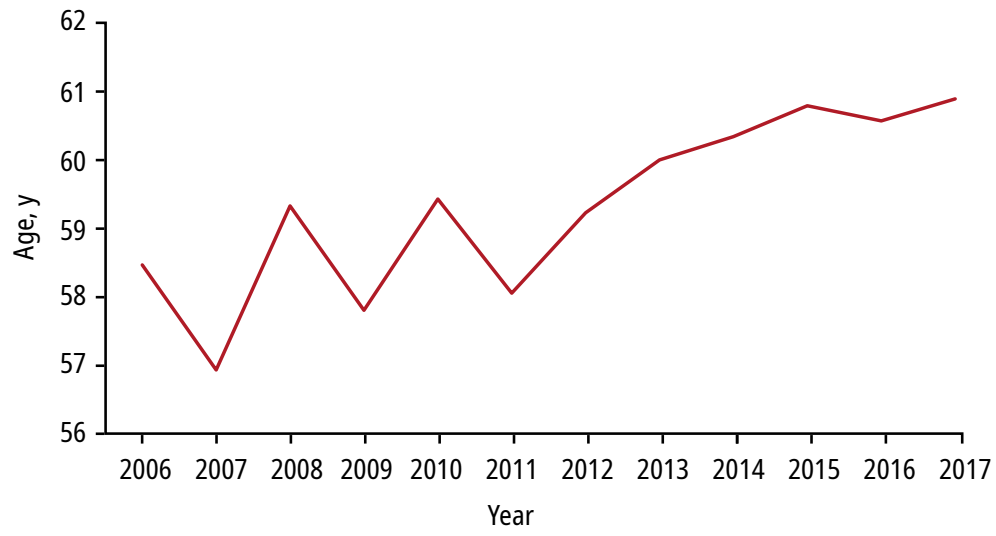

FIGURE 3 Mean age of patients undergoing pulmonary vein isolation or cavotricuspid isthmus ablation in-hospital stay has gradually decreased from 7.07 (4.11) days in 2006 to 4.91 (2.71) days in 2017 ( $P<0.005)$. A trend for the reduction of all-cause hospital readmissions rates $(55.8 \%$ to $25.4 \%$; $P<0.005$ ) in the 12 -month follow-up was reported, which was associated with a decrease in the number of hospital readmissions both due to cardiovascular disease $(P<0.005), \mathrm{AF}$ $(P<0.005)$, and heart failure $(P<0.005)$.

The 12-month follow-up data corroborated the fluctuations of annual rate of re-ablation with the highest value of $18.5 \%$ in 2007 and the lowest of $2.3 \%$ in 2006 ( $P<0.005)$. The proportion of patients who required cardioversion has decreased from $9.3 \%$ in 2006 to $3.2 \%$ in 2017 ( $P=0.005)$. Also, rates of all-cause mortality ( $2.3 \%$ in 2006 vs $0.2 \%$ in $2017 ; P<0.005)$, stroke ( $2.3 \%$ in 2006 vs $0.2 \%$ in $2017 ; P=0.047$ ), and MI (2.3\% in 2006 vs $0.4 \%$ in $2017 ; P=0.03$ ) decreased within 1 year following the index hospitalization.

DISCUSSION All patients from the SILCARD registry hospitalized between 2006 and 2017 with the main diagnosis of AF or AFL (ICD-10 code I48) who underwent transcatheter ablation procedure (ICD-9 procedures $37.341,37.342$, $37.272,37.261)$ were analyzed in this study. The initial population included 3201 patients, and 2745 participants remained for further follow-up after applying the follow-up criteria. The SILCARD database of catheter ablation for 
AF / AFL showed an increasing prevalence of comorbidities, arterial hypertension, and patients with a cardiac implantable electronic device between 2006 and 2017. Of note, data showed a gradual decrease of the length of in-hospital stay with a reduction in the number of readmissions and gradual improvement of outcomes in the 12-month follow-up period, reflected by lower rates of MI, stroke, and mortality (TABLE3). Data from the SILCARD database shows a rapid increase in the availability of catheter ablation for the treatment of AF/AFL in the population of Upper Silesia.

Upper Silesia is a highly urbanized region with a population of 4.57 million, including 3.8 million adults. In 2017, the ratio of $109 \mathrm{AF}$ ablation procedures per 1 million of inhabitants was reported, which is very similar to the average for whole Poland (114/million); however, much lower than in the West Pomerania Province (196/million), Mazovia Province (196/million), or Opole Province (181/million). In 2017, 87 electrophysiology laboratories were recognized in Poland, out of which 69 (79\%) performed PVI procedures. Only 5 of them are localized in Upper Silesia despite the fact that it is the second most populated region in Poland (approximately $12 \%$ of total population). The number of PVI procedures in Poland is growing every year, but still there is room for improvement in comparison with European countries most actively performing ablations like Germany, Denmark, Norway (according to the Supplement to the European Heart Rhythm Association's White Book). An overt explanation of these differences is the enormous disparity in gross domestic product (GDP) and health care expenditure among European Society of Cardiology (ESC) members. In the group of ESC members from central Europe, the highest mean number of PVI procedures adjusted for overall population count is found in the Czech Republic. ${ }^{11}$ Yet, in many western and northern European countries with high GDP, especially in the last couple of years, stagnation or even deceleration in the growth of the number of catheter ablation procedures was observed. On the other hand, there is a group of countries that, despite a relatively low GDP, enabled significant development of modern electrophysiology including AF management by catheter ablation.

Over 11 years of the SILCARD registry, an increase of more than 11-fold in the number of patients undergoing $\mathrm{AF}$ ablation (cryoballoon ablation or radiofrequency ablation) was recorded, from 43 in 2006 to 507 in 2017. This evolution is compatible with the natural trend observed worldwide. Although the number of ablations is constantly growing, it does not seem to fully cover the needs of rapidly ageing populations.

The management of AF by catheter ablation is significantly more efficient in reducing arrhythmia recurrence and improving quality of life when compared with the standard drug therapy, which was shown in big randomized trials. ${ }^{14,15}$ Circumferential PVI procedures, although pretty novel, have already marked their stable position in the strategy of rhythm control, and are constantly gaining in importance, which translates into the rise of heart centers equipped with electrophysiology laboratories, staff experience, and widening range and volume of ablation procedures.

A significant reduction in hospitalization time due to AF ablation in Upper Silesia was identified from 7 days in 2006 to less than 5 days in 2017, and also the incidence of prolonged hospital stays was significantly lower (lasting at least 7 days). This phenomenon may be attributed to increasing staff experience and performance, resulting in a substantial reduction of early complications requiring longer follow-up and additional treatment. During the follow-up period, there was a moderate, yet significant, growth of the average age of ablated patients from 58.5 years in 2006 to 60.9 years in 2017.

Similar to the registries from the United States, statistically more ablation procedures were performed in men; however, during the follow-up, there was a significant increase in the ratio of women to men from $27.9 \%$ in the beginning to $35.3 \%$ at the end of the study.

The analysis of patient comorbidities showed that with time, the burden of arterial hypertension in the ablated population was essentially increasing, but the incidence of stable coronary artery disease was decreasing. The percentage of patients with AF and a history of MI or ischemic stroke referred for PVI procedure was at a similar level with occasional pronounced fluctuations. Also, there were no evident differences in the incidence of congestive heart failure, type 2 diabetes mellitus, dyslipidemia, chronic kidney disease, or chronic obstructive pulmonary disease.

It is worth mentioning that when comparing characteristics of the populations submitted to the PVI procedure in different European countries, arterial hypertension, hyperlipidemia, and smoking rates were much higher in central European countries, including the $\mathrm{Czech}$ Republic and Poland. ${ }^{12}$

A substantial increase in representation of patients with pacemaker was visible, with highest numbers between 2008 and 2011. A similar pattern was identified in patients with CRT but the trend was slightly shifted in time, peaking between 2009 and 2014. No significant differences in the subgroup of patients with implantable cardioverter-defibrillator was detected. Tachyarrhythmia in the course of an $\mathrm{AF}$ episode can cause inadequate device interventions with high energy shocks. In CRT patients, it results in the loss of atrial function as well as ventricular synchronization. 
That is why the removal of the atrial substrate of the arrhythmia by catheter ablation remains an important element of recovering biventricular synchronization and bringing back the chance of becoming an optimal CRT responder. Thus, PVI represents one of the mainstays of sinus rhythm maintenance therapy in patients with depressed left ventricular systolic function..$^{16}$

During the entire follow-up period, only 1 in-hospital death related to the ablation procedure was recorded. The 12-month follow-up of all ablated patients revealed a significant decline in all-cause hospital readmissions (from 55.8\% in 2006 vs $25.4 \%$ in 2017), secondary to a substantial reduction in overall cardiovascular hospitalizations (44.2\% in 2006 vs $16.2 \%$ in 2017) as well as hospitalizations caused by AF recurrences (23.3\% in 2006 vs $9.7 \%$ in 2017) and heart failure onset or heart failure worsening (7\% in 2006 vs 3.4\% in 2017). Again, this is presumably the result of the increasing experience of the electrophysiology staff as well as improvements in the ablation method with an evident boost in efficiency as well as patient safety. The natural consequence of these factors is the rise of the ratio of patients free from arrhythmia incidents (or at least a significant reduction of episodes) and drop of the procedure-related late complications and adverse events. A huge step forward has been taken with fast modernization and improvement of the 3D mapping systems (CARTO 3, EnSIte Velocity, then Precision), standard use of irrigated catheters (since 2008), introduction of the cryoballoon ablation systems, ${ }^{17,18}$ and radiofrequency catheters allowing energy delivery under the control of constant contact force measurement (SmartTouch, Tacticath) $)^{19,20}$ and ultra-high-density substrate mapping. ${ }^{21}$ The optimization of periprocedural oral anticoagulants and the withdrawal of heparin bridging contributed to the reduction of early bleeding and thromboembolic adverse events.

Advancements in ablation techniques shifted the pattern of funds distribution, which was reported in a Canadian study by Samuel et al. ${ }^{22}$ Previously rising demand for medical resources and interventions in a population with $\mathrm{AF}$ changed dramatically after the index ablation procedure, resulting in an evident decline in healthcare resource utilization. ${ }^{23,24}$

Although the rate of patients requiring re-ablation because of the recurrence of AF fluctuated during 11 years of follow-up, peaking in 2007 and reaching a relative plateau between 2009 and 2014, a falling trend became settled since 2015 to the end of the follow-up period. Also, the need for additional cardioversion in the post-ablation group was clearly decremental through the entire study, which, again, is the success rate marker of arrhythmia elimination.

Despite the low initial incidence of MI in the 12-month post-procedural follow-up in the study population, a further decrease was observed. An explanation of this finding could be that an improvement in the procedure and success rate caused substantial eradication of the AF incidents or at least shortening of the episodes with clinically significant tachyarrhythmia(which is usually accompanied by a rise in high-sensitivity troponin concentrations) and lowering the probability of MI type 2 diagnosis.

Also, a reduction in all-cause mortality in the 1-year follow-up period in the postablation group was also observed. Global analyses performed by the ESC revealed that Poland, similar to other central European countries, declared low rate of cardiovascular complications when compared with the northern and western region ( $1.5 \%$ vs $4.7 \%$ ), but there were no evident differences in the occurrence of noncardiovascular adverse events. ${ }^{11,12}$

Study limitations The study is based on the electronic database of a single healthcare provider and it is limited to core variables, such as demographic data, comorbidities, length of in-hospital stay, and in-hospital morbidity and mortality. It does not cover data on laboratory results, echocardiographic parameters and pharmacotherapy, which represents a major limitation of the study. The quality of data is challenged by the discrepancy between the quality of data reporting by different centers and reimbursement bias. Due to the fact that a certain but small proportion of patients underwent cavotricuspid ablation for AFL with the use of 3D electro-anatomical mapping, the data should be interpreted with caution. This proportion was estimated based on the local data from the Upper-Silesian Medical Center in Katowice, which showed that cavotricuspid isthmus ablation for AFL with 3D mapping was performed in about $8.5 \%$ of patients.

Conclusions The study has shown a considerable increase in the number of catheter ablations for AF and AFL within the 12-year follow-up period in a highly populated urban area located in central Europe, reflecting the progress in cardiovascular healthcare. The efficacy of the procedure and clinical outcomes have incrementally improved. Taking into account the current indication for the procedure, the rate of procedure per million inhabitants still indicates its underutilization.

\section{ARTICLE INFORMATION}

CONFLICT OF INTEREST None declared

OPEN ACCESS This is an Open Access article distributed under the terms of the Creative Commons Attribution-Non Commercial-No Derivatives 4.0 International License (CC BY-NC-ND 4.0), allowing third parties to download articles and share them with others, provided the original work is properly cited, not changed in any way, distributed under the same license, and used for 
noncommercial purposes only. For commercial use, please contact the journal office at kardiologiapolska@ptkardio.pl.

HOW TO CITE Faryan M, Buchta P, Kowalski 0, et al. Temporal trends in the availability and efficacy of catheter ablation for atrial fibrillation and atrial flutter in a highly populated urban area. Kardiol Pol. 2020; 78: 537-544. doi:10.33963/ KP.15275

\section{REFERENCES}

1 Heeringa J, van der Kuip DA, Hofman A, et al. Prevalence, incidence and lifetime risk of atrial fibrillation: the Rotterdam study. Eur Heart J. 2006; 27: 949-953.

2 Andersson T, Magnuson A, Bryngelsson IL, et al. All-cause mortality in 272,186 patients hospitalized with incident atrial fibrillation 1995-2008: a Swedish nationwide long-term case-control study. Eur Heart J. 2013; 34: 1061-1067.

3 Roy D, Talajic M, Nattel S, et al; Atrial Fibrillation and Congestive Heart Failure Investigators. Rhythm control versus rate control for atrial fibrillation and heart failure. N Engl J Med. 2008; 358: 2667-2677.

4 Anter E, Callans DJ, Wyse DG. Pharmacological and electrical conversion of atrial fibrillation to sinus rhythm is worth the effort. Circulation. 2009; 120: 1436-1443.

5 Haïssaguerre $M$, Jaïs $P$, Shah $D C$, et al. Spontaneous initiation of atrial fibrillation by ectopic beats originating in the pulmonary veins. N Engl J Med. 1998; 339: 659-666.

6 Hindricks G, Sepehri Shamloo A, Lenarczyk R, et al. Catheter ablation of atrial fibrillation: current status, techniques, outcomes and challenges. Kardiol Pol. 2018: 76: 1680-1686.

7 Mark DB, Anstrom KJ, Sheng S, et al; CABANA Investigators. Effect of Catheter Ablation vs Medical Therapy on Quality of Life Among Patients With Atrial Fibrillation: the CABANA Randomized Clinical Trial. JAMA. 2019; 321: 1275-1285.

8 Ganesan AN, Shipp N], Brooks AG, et al. Long-term outcomes of catheter ablation of atrial fibrillation: a systematic review and meta-analysis. J Am Heart Assoc. 2013; 2: e004549.

9 Marrouche NF, Brachmann J, Andresen D, et al; CASTLE-AF Investigators. Catheter ablation for atrial fibrillation with heart failure. N Engl J Med. 2018; 378: 417-427. 10 Lenarczyk R, Mitręga K, Mazurek M, et al. Polish and European management strategies in patients with atrial fibrillation. Data from the EURObservational Research Programme-Atrial Fibrillation General Registry Pilot Phase (EORP-AF Pilot). Pol Arch Med Wewn. 2016; 126: 138-148.

11 Raatikainen MJP, Arnar DO, Merkely B, et al. A decade of information on the use of cardiac implantable electronic devices and interventional electrophysiological procedures in the European Society of Cardiology countries: 2017 report from the European Heart Rhythm Association. Europace. 2017; 19 (suppl 2): ii1-iigo. 12 Riahi $S$, Arbelo E, Brugada J, et al. Regional differences in referral, procedures, and outcome after ablation for atrial fibrillation in Europe: a report from the Atrial Fibrillation Ablation Pilot Registry of the European Society of Cardiology. Europace. 2016; 18: 191-200.

13 Gąsior M, Pres D, Wojakowski W, et al. Causes of hospitalization and prognosis in patients with cardiovascular diseases. Secular trends in the years 2006-2014 according to the SILesian CARDiovascular (SILCARD) database. Pol Arch Med Wewn. 2016; 126: 754-762.

14 Forleo GB, Mantica M, De Luca L, et al. Catheter ablation of atrial fibrillation in patients with diabetes mellitus type 2: results from a randomized study comparing pulmonary vein isolation versus antiarrhythmic drug therapy. J Cardiovasc Electrophysiol. 2009; 20: 22-28.

15 Wazni OM, Marrouche NF, Martin DO, et al. Radiofrequency ablation vs antiarrhythmic drugs as first-line treatment of symptomatic atrial fibrillation: a randomized trial. JAMA. 2005; 293: 2634-2640.

16 Pruszkowska P, Lenarczyk R, Gumprecht J, et al. Cryoballoon ablation of atrial fibrillation in patients with advanced systolic heart failure and cardiac implantable electronic devices. Kardiol Pol. 2018; 76: 1081-1088.

17 Fürnkranz A, Bordignon S, Dugo D, et al. Improved 1-year clinical success rate of pulmonary vein isolation with the second-generation cryoballoon in patients with paroxysmal atrial fibrillation. J Cardiovasc Electrophysiol. 2014; 25: 840-844.

18 Liu XH, Gao XF, lin CL, et al. Cryoballoon versus radiofrequency ablation for persistent atrial fibrillation: a systematic review and meta-analysis. Kardiol Pol. 2020; 78: 20-29.

19 Reddy VY, Dukkipati SR, Neuzil P, et al. Randomized, Controlled Trial of the Safety and Effectiveness of a Contact Force-Sensing Irrigated Catheter for Ablation of Paroxysmal Atrial Fibrillation: Results of the TactiCath Contact Force Ablation Catheter Study for Atrial Fibrillation (TOCCASTAR) Study. Circulation. 2015; 132: 907-915.

20 Kimura M, Sasaki S, Owada S, et al. Comparison of lesion formation between contact force-guided and non-guided circumferential pulmonary vein isolation: a prospective, randomized study. Heart Rhythm. 2014; 11: 984-991.

21 Wójcik M, Konarski Ł, Błaszczyk R, et al. High-density bipolar voltage mapping for substrate-guided ablation of atrial fibrillation. Kardiol Pol. 2018; 76: 1115.

22 Samuel M, Avgil Tsadok M, Joza J, et al. Catheter ablation for the treatment of atrial fibrillation is associated with a reduction in health care resource utilization. J Cardiovasc Electrophysiol. 2017; 28: 733-741.

23 Vamos M, Cappato R, Marchlinski FE, et al. Efficacy and safety of rivaroxaban compared with vitamin $\mathrm{K}$ antagonists for peri-procedural anticoagulation in catheter ablation of atrial fibrillation: a systematic review and meta-analysis. Europace. 2016; 18: 1787-1794.

24 Hohnloser SH, Camm AJ. Safety and efficacy of dabigatran etexilate during catheter ablation of atrial fibrillation: a meta-analysis of the literature. Europace. 2013; 15: 1407-1411. 